\title{
Metodología para la determinación de niveles de infestación por Cerambyx cerdo (Linnaeus, 1758), desarrollo de técnicas de captura y estudio de fauna saproxílica acompañante en los encinares de Mallorca
}

\author{
Núñez. L. ${ }^{1}$, Closa S. ${ }^{1}$, González, E. ${ }^{*}$, Lencina, J.L.,, Gallego, D. ${ }^{3,4}$ \\ ${ }^{1}$ Servei de Sanitat Forestal D.G. Espais Naturals i Biodiversitat, Conselleria de Medi Ambient, Agricultura i Pesca \\ (Govern de les Illes Balears). C/ Gremi de Corredors, $n^{\circ}$ 10, $1^{\circ}$, Poligono de Son Rossinyol, \\ 07009 - Palma- Illes Balears Tel.: 9711766 66, \\ ${ }^{2}$ SILCO, S.L. $n^{\circ} 12$ B, esc. 3, $2^{\circ}$ D, 28440, Guadarrama, Madrid \\ ${ }^{3}$ Departamento de Zoología y Antropología Física, Universidad de Murcia, \\ Campus Universitario de Espinardo. 30100 Murcia. \\ ${ }^{4}$ Sanidad Agrícola ECONEX, S.L., C/ Mayor, $n^{\circ} 15 B$ - Edificio ECONEX \\ Apartado de Correos $N^{\circ}$ 167, 30149 Siscar-Santomera, Murcia. \\ *Autor para correspondencia: lalogonzalezrosa@gmail.com
}

\section{Resumen}

Cerambyx cerdo Linnaeus, 1758 es un insecto perforador que afecta a los encinares de la isla de Mallorca. Sus daños son especialmente preocupantes en muchas zonas, donde afecta a buena parte de las encinas, prácticamente sin discriminación en cuanto a diámetro o edad del árbol. Se realiza una prospección de daños en toda la masa del encinar de la isla donde puede observarse su distribución, abundancia, así como sus efectos sobre el arbolado. Se ha creado una metodología que pretende servir de base para futuros muestreos, obteniéndose un mapa de afectación de Mallorca 2009 y se han prospectado con más detalle algunos montes públicos como Menut. Se han realizado trampeos con diferentes atrayentes durante varios años, lo que ha confirmado que el estatus de protección actual se ha de modificar y se ha propuesto declararlo plaga en Mallorca. Estos trabajos han servido de apoyo para la redacción de un proyecto de control en montes públicos con diferentes actuaciones para verificar cómo evolucionan cada una de ellas en el tiempo. Los trampeos han permitido conocer la fauna de coleópteros saproxílicos del encinar mallorquín.

Palabras clave: Daños, Islas Baleares, Encinas, Cerambyx cerdo. 


\section{Introducción}

La presencia de árboles añosos, así como decadentes, en el monte, desempeñan un rol primordial en el mantenimiento de las poblaciones de insectos saproxílicos. Este grupo de insectos son actores del proceso de descomposición de la madera (Micó E. et al. 2010), manteniendo los ciclos de materia y energía en el ecosistema forestal. Ahora bien, las prácticas selvícolas, especialmente las que han sido mal ejecutadas, permiten la proliferación de algunas especies de insectos que pueden causar desequilibrios indeseables en el ecosistema. En esta línea, masas forestales aprovechadas en el pasado, cuya selvicultura se ha abandonado y que han conformado una masa forestal envejecida son especialmente vulnerables a estos desequilibrios.

Los encinares mallorquines se encuentran ampliamente distribuidos por la Sierra de Tramuntana, espacio natural declarado Patrimonio de la Humanidad por la UNESCO en junio de 2011. Su utilidad medioambiental, económica y de uso público, es primordial para Mallorca. Los antiguos aprovechamientos para carbón, el envejecimiento de las cepas y el sobrepastoreo, están comprometiendo el desarrollo futuro de dichas masas forestales. En este escenario, Cerambyx cerdo se ha convertido en un agente acelerador del proceso de decaimiento del encinar, especialmente en aquellas zonas donde se ha abandonado la selvicultura tras una época de intensos aprovechamientos.

C. cerdo es un xilófago de distribución euroasiática, habitante de robledales y encinares íbero-baleares (Vives, 2001). Sus larvas se alimentan en el leño de los árboles provocando la reducción de la resistencia biomecánica de la madera, pudiendo llegar a la fractura de grandes ramas, acarreando la muerte de los árboles. Su actividad en el interior del árbol oscila entre los tres y los cinco años (Moral et al., 2010). Los adultos vuelan desde el mes de mayo siguiente al de su pupación hasta el mes de septiembre. Se emergencia se detecta por la aparición de grandes orificios elípticos en la corteza del árbol colonizado, así como por la acumulación de serrín en diversas zonas, habitualmente en la base del fuste (Núñez, 2004).

Aunque este coleóptero es una especie protegida por normativa internacional (Anexo II del Convenio de Berna de 1979, Anexo IV de Directiva de Hábitats), los seguimientos y estudios realizados en Mallorca desde el año 2008 muestran la presencia habitual de $C$. cerdo y su relación con daños irreparables en numerosas masas de encinar (González et al., 2010). Recientes documentos consideran la necesidad de variar el estatus de protección de esta especie, excluyéndola de la Directiva Hábitats (Viñolas y Vives, 2010).

Desde el Servicio de Sanidad Forestal del Gobierno de las Islas Baleares ya se ha planteado este problema desde hace tiempo (Núñez, 2002), y se pretende encontrar soluciones a un deterioro que aumenta año tras año, poniendo en peligro la sostenibilidad de los encinares mallorquines, que ocupan 26.549 ha, según el tercer Inventario Forestal Nacional y que constituyen el bosque más característico de Mallorca.

Por lo tanto, es indispensable la evaluación de niveles de afección por $C$. cerdo en las masas de encinar mallorquinas, no sólo para conocer el alcance de los daños de este insecto y evaluar la necesidad de llevar a cabo actuaciones de control, sino 
también para detectar aquellas zonas que se encuentren en peores condiciones y poder realizar un manejo fitosanitario eficaz. Las evaluaciones de niveles de daño por C.cerdo en muchos montes de encinar en Mallorca justifican la necesidad de reducir población de este insecto. En este sentido, desde el año 2008 se han testado diferentes modelos de trampa y atrayentes. En este trabajo se presentan resultados sobre los ensayos para la determinación de los niveles de infestación, el desarrollo de métodos de captura, y el estudio de fauna saproxílica acompañante de C.cerdo en los encinares de Mallorca.

\section{Material y métodos}

Para evaluar los daños en masas de encinar se elaboraron mallas que determinaron los puntos a muestrear. Para el mapa mallorquín se estableció una malla de 4 kilómetros de lado, mientras que para mapas de mayor detalle, como los de montes públicos, se usó un lado de malla de 200 metros. En cada punto de muestreo que define la malla se caracterizaron veinte encinas, en las que se tomaron datos tanto dasométricos como fitosanitarios. Se evaluó la presencia de C. cerdo mediante la observación de síntomas inequívocos asociados a la presencia de esta especie:

- Amontonamientos de serrín en la base del árbol, fuste u horcaduras de ramas.

- Orificios elípticos de emergencia, del tamaño adecuado, tanto en fuste como en base y ramas.

- Observación de ramas secas, síntoma frecuente cuando la infestación es muy elevada.

Las fichas de cada monte fueron procesadas para obtener el nivel de daño del árbol individual, y posteriormente el grado de infestación del punto de muestreo. El baremo utilizado para evaluar el árbol individual fue el siguiente:

Grado 0: Sin daños, árbol sano.

Grado 1: Sin daños actuales, acaso con daños antiguos visibles.

Grado 2: Con daño actual, leve o puntual; presencia de algún síntoma específico de C. cerdo, como montones de serrín u orificios de emergencia.

Grado 3: Con daño actual, generalizado o más severo. Presencia en el mismo pie de abundantes síntomas de los anteriormente descritos.

Grado 4: Árbol muerto o moribundo, con daños frecuentes propios de C. cerdo.

Para la gradación de niveles de infestación del punto de muestreo se definieron seis niveles de infestación:

Nivel 0: Sin daños. Población sana. Todos o casi todos los pies con grado 0.

Nivel 1: Presente. Algunos daños esporádicos. El $25 \%$ de los pies con daños en grado bajo: 0,1 ó a lo sumo grado 2 .

Nivel 2: Frecuente. Hay daños, pero no son excesivos. Menos del $50 \%$ de los pies con daño, y nunca en grado 3. 
Nivel 3: Abundante. Muchos daños. El $50 \%$ ó más de los pies presentan daños. Hay pies con grado 3.

Nivel 4: Grave. Hay árboles muertos y/o decadentes. Más del $50 \%$ de los pies presentan daño, y algunos de ellos adquieren grado 4.

Nivel 5: Muy grave. Daños severos. Al menos el $75 \%$ de los pies inventariados presentan daños, siendo los grados 3 y 4 abundantes.

De forma práctica también se han utilizado niveles intermedios a los descritos.

\section{Desarrollo de técnicas de captura}

Desde el año 2008 se han instalado trampas en diferentes montes públicos. Como cebo se utilizó el atrayente cairomonal desarrollado por Sánchez et al. (2007) y comercializado como Econex Cerambyx (Econex, Murcia) (). Como trampa se ha utilizado una trampa flexible que permite el ajuste al tronco de la encina donde ha de ser instalada, la trampa Crosstrap ${ }^{\circledR}$ Xilófagos (Econex, Murcia).

\section{Estudio de fauna saproxílica acompañante}

Además de ejemplares de C. cerdo, también se han capturado otros insectos, en su mayor parte coleópteros. Para la determinación de las especies de la comunidad de saproxilófagos se ha llevado a cabo la siguiente metodología: las muestras de campo eran conservadas en viales en etanol al 70\% y transportados a laboratorio. Las muestras se limpiaron bajo agua corriente, y se realizó el recuento de todos los individuos, identificando cada especie con ayuda de lupa binocular. En caso necesario se recurrió a la extracción y examen de la genitalia masculina o femenina para completar la determinación.

\section{Resultados}

Las evaluaciones de los niveles de infestación han permitido la obtención de mapas de daño con alto grado de detalle como el de la finca Menut (Figura 1) o el mapa de infestación del contiguo monte de Binifaldó, localizado también en el término municipal de Escorca.

En la tabla 1 pueden observarse los niveles de captura del coleóptero, relacionados con los niveles poblacionales que alcanza $C$. cerdo en el monte de Menut. En ella se observan evidentes variaciones interanuales de los resultados, así como las fechas de actividad del insecto.

En los trampeos realizados desde 2010 se han contabilizado hasta 100 taxones de coleópteros, 61 de los cuales se han determinado a nivel específico. Entre ellas han aparecido los siguientes endemismos baleáricos: Cryptocephalus (Burlinius) majoricensis De la Fuente 1918 (Familia Chrysomelidae), Nacerdes (Xanthochroa) raymondi canyellesi Vázquez 2006 (Fam. Oedemeridae) y Misolampus goudoti Gue- 


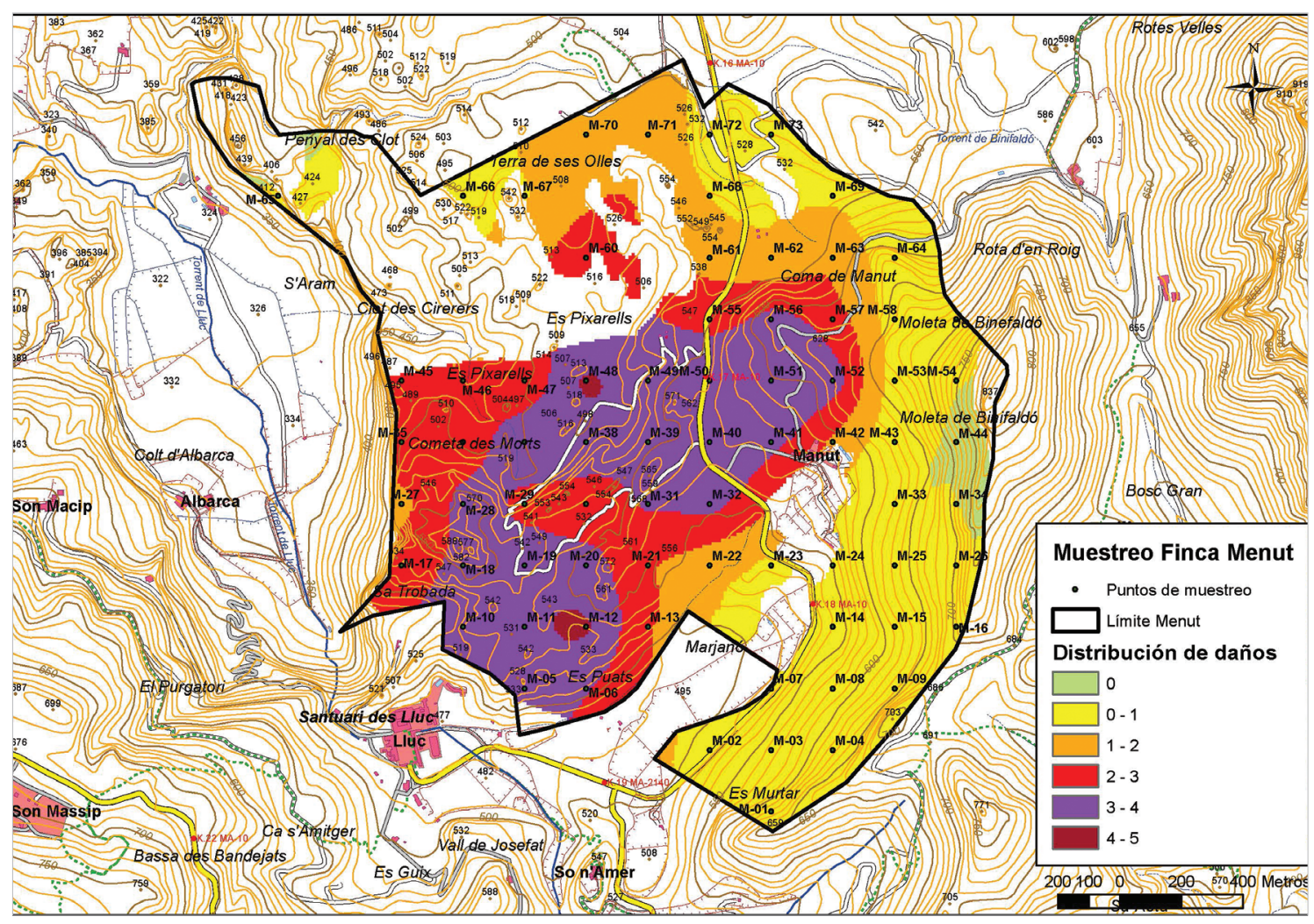

Figura 1. Mapa de niveles de infestación del monte de Menut (Escorca, Mallorca).

Tabla 1. Capturas de C. cerdo en veinte trampas instaladas en Menut (Escorca, Mallorca) en el periodo 2010-2015.

\begin{tabular}{|r|r|r|r|r|r|r|r|c|}
\hline FECHA & 7 jun & 17 jun & 23 jun & 7 jul & 22 jul & 4 ago & 16 ago & TOTAL \\
\hline \hline AÑO 2010 & - & INSTAL. & 0 & 75 & 183 & 25 & 11 & 294 \\
\hline \hline AÑ̃ 2012 & - & INSTAL. & 38 & 137 & 23 & 0 & 0 & 198 \\
\hline \hline AÑO 2014 & - & INSTAL. & 16 & 92 & 13 & 0 & 6 & 127 \\
\hline \hline AÑO 2015 & INSTAL. & 16 & 18 & 217 & 23 & 4 & 0 & 278 \\
\hline
\end{tabular}

rin-Meneville 1834 (Fam. Tenebrionidae) Además, se han capturado dos nuevas citas para Baleares: Colydium elongatum Fabricius, 1787 y Synchita fallax Schuh 1998 (ambos de la fam. Zopheridae).

Durante el trampeo de 2012 se capturaron 74 taxones de coleópteros, de los que 55 de ellos han sido identificados a nivel específico y entre los cuales vuelve a aparecer abundantemente el endemismo Nacerdes (Xanthochroa) raymondi canyellesi lo que ratifica la presencia de poblaciones estables del insecto en estos montes. Otro insecto capturado en 2012 de forma frecuente es el Zopheridae Synchita fallax Schuh 1998. Salvo para el caso de Cerambyx cerdo y Trichoferus holosericeus Rossi 1790 
(particularmente abundante) no se han obtenido niveles elevados de ninguna otra especie de la familia Cerambycidae.

\section{Discusión}

Los trabajos de evaluación de niveles de infestación, así como los trampeos que se realizan tanto en masas de encinar muy afectadas como en otras no tan afectadas, muestran que las poblaciones de $C$. cerdo en los encinares de Mallorca son muy elevadas. Paradójicamente, $C$. cerdo es una especie protegida en el anexo II de la Directiva 97/62/CE como "especie de interés comunitario", por la IUCN que en 1996 la consideró "especie vulnerable”, así como en el anexo II del Convenio de Berna como "especie estrictamente protegida", si bien en su transposición a la legislación nacional figura con menor grado de protección. Por otro lado, los bosques esclerófilos mediterráneos cuya especie vegetal dominante es Quercus ilex, figuran entre los Hábitats naturales de interés comunitario, incluidos en la Directiva Hábitats 92/43/CEE del Consejo (código "9340 bosques de Quercus ilex y de Quercus rotundifolia"). Esto constituye una nueva paradoja, puesto que la acción de C.cerdo pone en peligro la conservación de estas masas. Urge por tanto reconsiderar el estatus de protección que tiene esta especie, al menos en el sur de Europa.

A los problemas que ocasiona $C$. cerdo en los encinares mallorquines, se suman los causados al regenerado debidos a la presión de los herbívoros. Es especialmente preocupante que la regeneración por semilla no sea suficiente para garantizar la sostenibilidad futura de estas masas.

La notable diversidad de coleópteros saproxílicos registrados confiere a estos dispositivos de trampeo una importante utilidad para el conocimiento de la entomofauna que habita las masas de encinar de Mallorca, y para su utilización en diversos aspectos de su gestión forestal.

\section{Conclusiones}

Se ha conseguido diseñar una metodología para la evaluación de niveles de infestación del insecto perforador $C$. cerdo. Su uso ayudará a la gestión selvícola de las masas de encinar. La aplicación de este método en montes públicos ha permitido hasta la fecha detectar niveles de afección muy elevados, particularmente en aquellas zonas donde se realizaron antiguamente aprovechamientos de carbón.

El desarrollo de los métodos de captura desde el año 2008 ha permitido la comer-

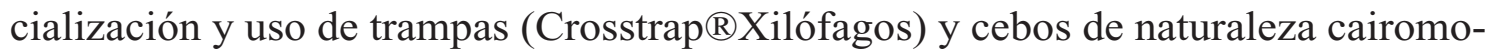
nal (Econex Cerambyx) muy eficientes para la captura de C. cerdo. Su uso continuado en las mismas zonas, cuando se trata de áreas muy afectadas, ha permitido estimar la elevadísima población de C. cerdo, en coherencia con estudio de los daños.

Se han determinado más de sesenta especies de coleópteros asociados a C. cerdo en los lugares de trampeo. Entre ellas destacan algunos endemismos baleáricos, así 
como citas nuevas para Mallorca. Muchos de estos insectos, presentan poblaciones estables en los encinares mallorquines. Esta información es muy útil de cara a la gestión futura de estas masas.

\section{Bibliografía}

González, E., Gallego, D., Lencina, J.L., Closa, S., Muntaner, A. y Núñez, L., 2010. Propuesta de una metodología para la determinación de los niveles de infestación por Cerambyx cerdo (Linnaeus, 1758) (Coleoptera: Cerambycidae). Evaluación de los niveles de infestación en Mallorca, año 2009. Boletín de Sanidad Vegetal-Plagas, 2010, 2: 157-163.

Micó, E., Marcos-García, M.A., Quinto, J., Ramírez, A., Ríos, S., Padilla, A. y Galante, E., 2010. Los árboles añosos de las dehesas ibéricas, un importante reservorio de insectos saproxílicos amenazados. Elytron, 2010. Vol. 24: 89-97.

Moral, J., Casado, D., Gallego, M. y Rey, J.M., 1994. Presencia de insectos parásitos del grupo Cerambyx cerdo en la dehesa extremeña. Phytoma-España 59: 44-52.

Núñez, L., 2002. El banyarriquer. L insecte perforador que ataca als alzinars. Consellería de Medi Ambient de les Illes Balears. Quadern de natura núm. 14.

Núñez, L., 2004. El caso del gran capricornio o banyarriquer (Cerambyx cerdo, Linnaeus, 1758) en las Islas Baleares. Revista Foresta núm. 24. Colegio de Ingenieros Técnicos Forestales.

Sánchez, I., Domínguez, L., López, G., Ruiz, J.M., Rodríguez, S. y Carrasco, A., 2007. La incidencia, comportamiento y posibilidades de control de cerambícidos xilófagos de encina y alcornoque. XXIV Reunión Anual del Grupo de Trabajo Fitosanitario de Forestales, Parques y Jardines. Marbella (Málaga), noviembre de 2007.

Viñolas, A. y Vives, E., 2010. Cerambyx cerdo. En W. AA. 2010. Bases ecológicas preliminares para la conservación de las especies de interés comunitario en España: Invertebrados. Ministerio de Medio Ambiente y Medio Rural y Marino. Madrid. 63 pp.

Vives, E., 2001. Atlas fotográfico de los cerambícidos íbero-baleares (Coleoptera). Argania Editio, S.C.P., 287 pp. 
